\title{
Prevalência e fatores associados da disfunção sexual em mulheres após mastectomia: estudo transversal
}

Prevalence and associated factors of sexual dysfunction in women after mastectomy: a cross-sectional study Prevalencia y factores asociados de disfunción sexual en mujeres después de una mastectomía: estudio transversal Caroline Mendes dos SANTOS ${ }^{1}$

Gisely Fernandes da SILVA ${ }^{\mathbf{1}}$

Mateus Dias ANTUNES ${ }^{2}$

Michelle Cardoso Machado dos SANTOS ${ }^{1}$

Paulo TSUNETA ${ }^{1}$

Kelley Cristina COELHO ${ }^{1}$

${ }^{1}$ Universidade Cesumar (UniCesumar), 87050-900 Maringá - PR, Brasil

${ }^{2}$ Faculdade de Medicina da Universidade de São Paulo (USP), Departamento de Fisioterapia, Fonoaudiologia e Terapia Ocupacional FM/MFT, 05360-160 São Paulo - SP, Brasil

\section{Resumo}

O câncer de mama é um dos tumores mais incidentes entre as mulheres ocasionando acometimentos cardiovasculares, motores e sexuais. O objetivo do estudo foi identificar a prevalência de disfunção sexual em mulheres mastectomizadas e analisar a correlação entre os domínios da resposta sexual nas fases da resposta sexual feminina. Trata-se de um estudo transversal com 54 mulheres que realizaram a cirurgia de mastectomia com idades entre 30 a 59 anos. Para avaliar a disfunção sexual foi utilizado o instrumento Female Sexual Function Index. Foi possível verificar que a prevalência de disfunção sexual entre as mulheres mastectomizadas foi de $77,7 \%$. As fases da resposta sexual que mostraram-se significantemente afetadas foram: excitação $(r=0,86)$, lubrificação $(r=0,80)$, orgasmo $(r=0,86)$, satisfação $(r=0,81)$, dor $(r=0,80)$ e a primeira fase da resposta sexual que é o desejo $(r=0,41)$ apresentou correlação moderada. Em relação ao desempenho sexual, nenhuma mulher obteve desempenho excelente, somente 12 apresentaram resultado bom e 42 tiveram resultado ruim. Houve uma alta prevalência de disfunção sexual entre as mulheres mastectomizadas e apresentando alteração nos aspectos relacionados com a excitação, lubrificação, orgasmo, satisfação e dor. Sugerimos, portanto, que novos estudos que abordem essa temática sejam realizados, para elucidar a relação entre os domínios da resposta sexual e a disfunção sexual em mulheres submetidas à mastectomia. Fica evidente a importância de promover estratégias, ações e intervenções de promoção da saúde para mulheres mastectomizadas no contexto da interdisciplinaridade.

Descritores: Câncer de Mama; Saúde da Mulher; Promoção da Saúde.

\section{Abstract}

Breast cancer is one of the most common tumors among women, causing cardiovascular, motor and sexual disorders. The objective of the study was to identify the prevalence of sexual dysfunction in mastectomized women and to analyze the correlation between the domains of sexual response in the phases of female sexual response. This is a cross-sectional study with 54 women who underwent mastectomy surgery aged 30 to 59 years. To evaluate sexual dysfunction, the Female Sexual Function Index instrument was used. It was possible to verify that the prevalence of sexual dysfunction among mastectomized women was $77.7 \%$. The phases of sexual response that were significantly affected were: arousal $(r=0.86)$, lubrication $(r=$ $0.80)$, orgasm $(r=0.86)$, satisfaction $(r=0.81)$, pain $(r=0.80)$ and the first phase of the sexual response, which is desire $(r=$ 0.41 ), presented a moderate correlation. Regarding sexual performance, no woman had an excellent performance, only 12 had a good result and 42 had a bad result. There was a high prevalence of sexual dysfunction among mastectomized women, with changes in aspects related to arousal, lubrication, orgasm, satisfaction and pain. We suggest, therefore, that further studies addressing this theme should be carried out, to elucidate the relationship between the domains of sexual response and sexual dysfunction in women undergoing mastectomy. The importance of promoting health promotion strategies, actions and interventions for mastectomized women in the context of interdisciplinarity is evident.

Descriptors: Breast Cancer; Women's Health; Health Promotion.

\section{Resumen}

El cáncer de mama es uno de los tumores más comunes entre las mujeres y causa trastornos cardiovasculares, motores y sexuales. El objetivo del estudio fue identificar la prevalencia de disfunción sexual en mujeres mastectomizadas y analizar la correlación entre los dominios de la respuesta sexual en las fases de la respuesta sexual femenina. Se trata de un estudio transversal con 54 mujeres que se sometieron a cirugía de mastectomía de 30 a 59 años. Para evaluar la disfunción sexual se utilizó el instrumento Índice de Función Sexual Femenina. Se pudo comprobar que la prevalencia de disfunción sexual entre mujeres mastectomizadas fue del $77,7 \%$. Las fases de la respuesta sexual que se vieron significativamente afectadas fueron: excitación $(r=0,86)$, lubricación $(r=0,80)$, orgasmo $(r=0,86)$, satisfacción $(r=0,81)$, dolor $(r=0,80)$ y la primera fase de la respuesta sexual, que es el deseo $(r=0,41)$, presentó una correlación moderada. En cuanto al desempeño sexual, ninguna mujer tuvo un desempeño excelente, solo 12 tuvieron un buen resultado y 42 tuvieron un mal resultado. Hubo una alta prevalencia de disfunción sexual entre las mujeres mastectomizadas, con cambios en aspectos relacionados con la excitación, lubricación, orgasmo, satisfacción y dolor. Sugerimos, por tanto, que se realicen más estudios que aborden este tema, para dilucidar la relación entre los dominios de la respuesta sexual y la disfunción sexual en mujeres sometidas a mastectomía. Es evidente la importancia de promover estrategias, acciones e intervenciones de promoción de la salud para mujeres mastectomizadas en el contexto de la interdisciplinariedad.

Descriptores: Cáncer de Mama; La salud de la Mujer; Promoción de la Salud.

INTRODUÇÃO

O câncer de mama representa a neoplasia de maior prevalência entre as mulheres nos últimos anos. O Brasil tem uma média de 56 casos a cada 100 mil mulheres ${ }^{1}$. No entanto, as abordagens no tratamento do câncer apresentaram importantes avanços tecnológicos em diversas áreas, permitindo assim, uma maior expectativa e qualidade de vida $^{2}$.

Nos últimos anos, as possibilidades terapêuticas mais utilizadas para o tratamento de mulheres com câncer de mama é a quimioterapia, radioterapia, hormonioterapia e a mastectomia $^{3}$. De acordo com o Instituto 
Nacional do Câncer (INCA $)^{2}$, a modalidade de tratamento deve ser baseada na fase da doença.

A mastectomia é o tratamento mais utilizado em mulheres acometidas pelo câncer de mama, que visa a não recidiva do tumor, e esse procedimento cirúrgico objetiva a remoção de ambas ou uma das mamas, com remoção total do tumor ${ }^{2}$. A mastectomia radical realiza a retirada total da mama além de músculos próximos ao local, as mediações podem variar conforme a extensão do câncer na mama acometida ${ }^{4}$.

A autoaceitação da mulher mastectomizada diante das mudanças corporais submetidas recentemente é um processo difícil, complexo e relativamente demorado ${ }^{5}$ que interferem fortemente sobre a expressão e sexualidade feminina ${ }^{3}$. A disfunção sexual feminina (DSF) é responsável por alterar uma ou em várias fases da resposta sexual ${ }^{6}$ gerando quadros clínicos que envolvem a diminuição do desejo sexual, alterações na excitação sexual, diminuição da lubrificação, repulsão sexual, transtorno de orgasmo e condições dolorosas como dispareunia e vaginismo ${ }^{7-8}$.

Considerando ao fato que é durante 0 pós-tratamento que se manifestam de maneira mais evidente os impactos na área emocional e sexual 4 , todos esses fatores corroboram para o surgimento das disfunções sexuais e enfatiza a importância de melhorar as evidências científicas da área ${ }^{9-10}$. Dessa forma, o objetivo desse estudo é identificar a prevalência de disfunção sexual em mulheres mastectomizadas e analisar a correlação entre os domínios da resposta sexual nas fases da resposta sexual feminina.

\section{MATERIAL E MÉTODO}

Trata-se de estudo transversal, realizado no período de maio a junho de 2020 na Universidade Cesumar (UniCesumar), aprovado pelo Comitê de Ética em Pesquisa da Universidade Cesumar sob parecer $n^{\circ} 4052955$,

Foram considerados como critérios de inclusão: mulheres que tiveram câncer de mama e submetidas à cirurgia de mastectomia. Já como critérios de exclusão, foram considerados outros indivíduos que foram acometidos por outros tipos de cânceres originados de outras regiões do corpo.

O estudo foi composto por mulheres mastectomizadas, com idades entre 30 a 59 anos participantes da Rede Feminina de Combate ao Câncer dos municípios de Maringá e Astorga - Paraná, e da Clínica Escola de Fisioterapia da Universidade Cesumar.
Os procedimentos foram realizados de maneira virtual pela ferramenta Microsoft Forms. A divulgação eletrônica da pesquisa foi realizada por meio de um vídeo que constava sobre o objetivo da pesquisa e instruções sobre o preenchimento do instrumento. Após finalizarem a resolução do questionário online, todas as mulheres receberam uma cartilha confeccionada pelos autores, contendo orientações com diversos exercícios domiciliares de mobilidade para os membros superiores.

Todos as mulheres aceitaram participar do estudo do estudo, assinando o Termo de Consentimento Livre e Esclarecido, de acordo com a Resolução 196/96, segundo a determinação do Conselho Nacional de Saúde. Todas as mulheres foram orientadas sobre o objetivo do estudo e os passos para a sua realização.

Para avaliar a disfunção sexual, foi utilizado o Questionário "Female Sexual Function Index" (FSFI). Trata-se de um instrumento traduzido e validado para população brasileira, que apresenta 19 questões que envolvem a atividade sexual da mulher nas últimas quatro semanas. Ele é dividido em seis domínios que avaliam o grau de desejo, excitação, lubrificação, satisfação, orgasmo e dispareunia. O escore final utilizado para a classificação do desempenho sexual é obtido pela somatória de cada área multiplicado por um fator compatível e pode variar de no mínimo 2 a no máximo 36 pontos. É considerado classificado como "excelente" valores maiores de 36, "bom" valores entre 26,6 a 35,9 e "ruim" os valores menores de $26,5^{11}$.

Após a finalização do estudo, foi realizada a análise estatística dos dados com o software estatístico SPSS Statistics versão 26, por meio de uma análise estatística descritiva e as variáveis empregadas para analisar os dados foram a correlação linear de pearson $(r)$ e 0 teste de normalidade de Shapiro-Wilk.

RESULTADOS E DISCUSSÃO

Participaram do estudo 54 mulheres, com média de idade de 44,25 $\pm 8,27$ anos, similar aos dados levantados pelo INCA ${ }^{2}$ que destacam que a partir dos 40 anos ocorre aumento da prevalência de casos de câncer de mama entre as mulheres e os índices de mortalidade (Tabela 1). No presente estudo, observou-se que a prevalência de DSF entre as mulheres mastectomizadas foi de $77,7 \%$ o que corrobora com Canário et al. $^{12}$ que observou uma prevalência de DSF de $75 \%$ após mastectomia nas mulheres. Estudo de Sreelakshmy et al. ${ }^{13}$, 
apresentaram a prevalência de DSF mais elevada chegando a 90\%. Já em relação ao desempenho sexual, nenhuma das participantes do presente estudo apresentaram desempenho sexual excelente, $22,2 \%$ apresentaram desempenho sexual bom, e $77,7 \%$ apresentaram desempenho sexual ruim (Figura 1).

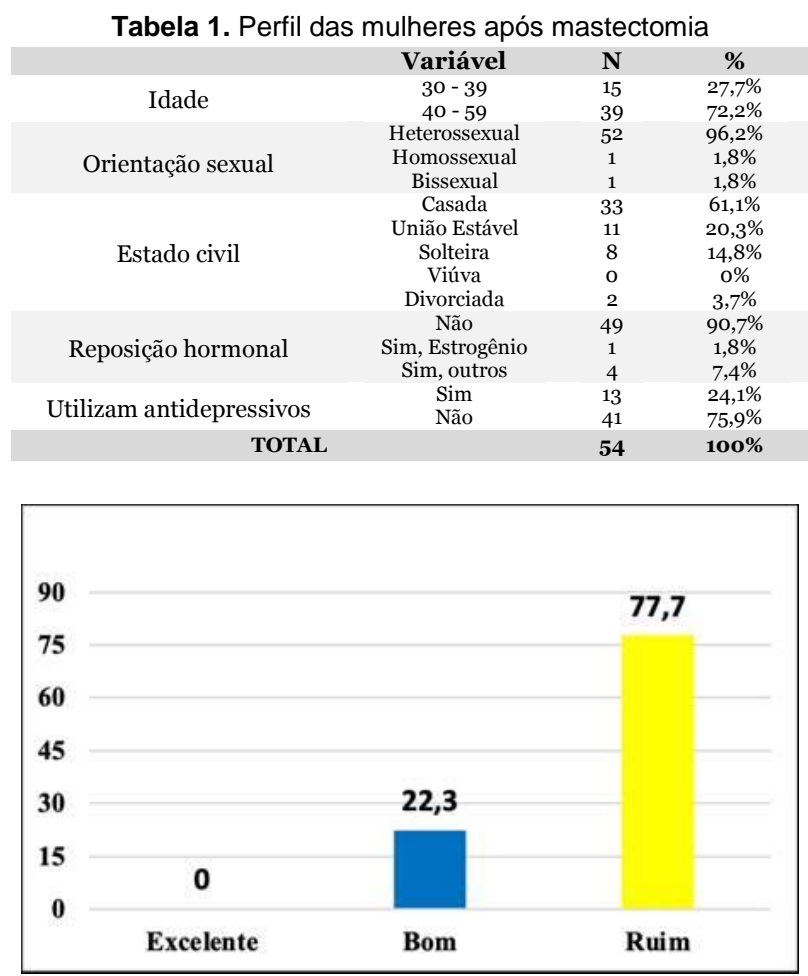

Figura 1: Percentual do desempenho sexual de mulheres após mastectomia.

A análise da correlação linear das fases da resposta sexual feminina está apresentada na Tabela 2. Os resultados apontam que as variáveis dos domínios que mostraram-se significantemente afetadas foram: excitação $(r=$ $0,86)$, lubrificação $(r=0,80)$, orgasmo $(r=0,86)$, satisfação $(r=0,81)$ e dor $(r=0,80)$, o que proporcionaram uma influência forte no escore individual de cada domínio respectivamente. Entretanto, a primeira fase da resposta sexual que é o desejo $(r=0,41)$, apresentou correlação moderada.

Tabela 2. Análise da correlação linear das Fases da Resposta Sexual Feminina em seus diferentes domínios de mulheres após mastectomia

\begin{tabular}{l|l|}
\hline Domínio & Valor de $\mathbf{~}$ \\
\hline Desejo & 0,41 \\
\hline Excitação & 0,86 \\
\hline Lubrificação & 0,80 \\
\hline Orgasmo & 0,86 \\
\hline Satisfação & 0,81 \\
\hline Dor & 0,80 \\
\hline
\end{tabular}

Legenda: Valores de 0,00 a 0,19 correlação bem fraca; 0,20 a 0,39 correlação fraca; 0,40 a 0,69 correlação moderada; 0,70 a 0,89 uma correlação bem forte; 0,90 a 1,00 correlação muito forte.

Os resultados obtidos são referentes a uma das questões de cada domínio de forma individual, não avaliando o domínio de forma geral, mas às questões a eles pertencentes que tiverem maiores índices de alterações. Logo, foi possível observar que $30 \%$ das mulheres relataram ter sentido poucas vezes desejo sexual, inclusive classificaram o desejo sexual como baixo ou ausente. Cenário este que contrasta do exposto no estudo de Pegorare et al. $^{14}$ em que $50 \%$ das participantes relataram nunca ou poucas vezes sentirem desejo sexual, no entanto ocorre uma taxa de $30 \%$ para libido baixo ou ausente, equiparando-se aos dados do atual estudo.

Dessa maneira, apenas 20,3\% afirmaram estar quase sempre satisfeitas com a excitação durante a atividade sexual e 33,3\% classificaram sua excitação como baixa ou ausente. Para fins analíticos, achados semelhantes foram relatados por Pegorare et al. ${ }^{14}$, em que mulheres classificam a excitação como baixa ou ausente, nesse sentido totalizouse a pontuação de $36,1 \%$.

Mediante 0 estudo supracitado, é inerente a pontuação de $27,7 \%$ das mulheres que não tiveram êxito em manter a lubrificação vaginal necessária durante 0 ato sexual. É evidente que assemelhando-se com os dados da presente pesquisa que mostrou que $22,2 \%$ das participantes tiverem dificuldade em manter a Lubrificação durante 0 ato sexual.

Ademais, somente $27,7 \%$ das mulheres, poucas vezes, conseguiram atingir o orgasmo, resultado este mostrado através de umas das questões referentes a este domínio. É interessante que, comparado com Pegorare et al. ${ }^{14}$ obteve-se um dado mais elevado em seu estudo em que as mulheres que nunca ou raramente atingiram o orgasmo chegou-se à porcentagem de $36,1 \%$. Ainda, referente à satisfação em nosso estudo, 35\% afirmaram estar muito insatisfeitas com suas vidas sexuais.

Para o conhecimento, em relação a dor, $20,3 \%$ das mulheres relataram quase sempre ou sempre ter dor ou desconforto durante a penetração vaginal. Nesse sentido, a dor é desencadeada pelos efeitos e consequências das drogas utilizadas durante o tratamento para - câncer de mama. Essas afirmações corroboram para explanar que, em suma, o tratamento leva algumas mulheres sentirem dor durante a relação sexual, um desses efeitos seria a menopausa precoce que ocasionará o ressecamento vaginal ${ }^{15}$.

Efetivamente, nota-se que apesar dessas mulheres apresentarem 0 desejo moderado, ou seja, interesse em iniciar o ato sexual, logo vem uma memória, a lembrança negativa referente a relação sexual dolorosa, interferindo nas demais etapas da relação em que a mesma não é bem sucedida. A sexualidade da mulher pode ser afetada 
independentemente do tipo de tratamento para o câncer de mama, ocasiona-se alterações, tanto psicológicas quanto físicas, que influenciam negativamente no que tange os domínios da função sexual ${ }^{15}$.

No presente estudo, mostrou-se que as mulheres solteiras após mastectomia tem uma prevalência maior de DSF sendo de 100\% quando comparadas às mulheres casadas $72,7 \%$. Entre as mulheres mastectomizadas que utilizam antidepressivos obtiveram uma prevalência de DSF de $84,6 \%$. Observa-se que as diversas excitações, a lubrificação, o orgasmo, a satisfação e a dor foram afetadas significativamente após mastectomia. De forma geral, em relação ao domínio do desejo manteve-se apenas uma correlação moderada. Já Lopes $^{16}$ relatou alteração significativa em todos os domínios como desejo, excitação, lubrificação, orgasmo e satisfação.

As mulheres do presente estudo com idade mais avançada apresentaram uma prevalência de DSF de $76,9 \%$, e as mulheres mais jovens apresentaram uma prevalência de $73,3 \%$, entretanto, tais dados contradiz-se com o estudo de Canário et al. ${ }^{12}$, que apresentou maior prevalência de DSF em mulheres mais jovens do que em mais velhas. Ademais, observou-se neste estudo uma porcentagem significativa de prevalência de DSF de 100\% entre as mulheres solteiras que utilizam antidepressivos. Souza ${ }^{16}$ enfatiza que os medicamentos antidepressivos contribuem para alterações da atividade sexual, esses afetam as fases do desejo, excitação e orgasmo ${ }^{17}$.

Segundo Araújo et al. ${ }^{18}$, as mulheres que apresentam DSF podem ter depressão moderada ou grave, sendo 13,5 vezes maior do que nas mulheres que não possui DSF. Porém como em nosso estudo a amostra de mulheres que utilizam antidepressivos foi baixa, sendo de somente 8 mulheres, assim torna-se necessário estudos futuros e especificamente voltados a essa temática para um levantamento de dados com maior precisão e confiabilidade.

Sendo assim, o tratamento das DSF deve ser de caráter multidisciplinar, visto que, as causas que levam as alterações na resposta sexual fisiológica são multifatoriais ${ }^{19}$. Nesse sentido, seja qual for a fase em que isso ocorra na vida da mulher, as consequências da cirurgia de retirada da mama são de grande impacto, sobretudo na alteração total da rotina para quem está passando pelo processo e também para os familiares ${ }^{3}$.

O presente estudo apresentou algumas limitações, a principal é o número pequeno de participantes e ausência de um cálculo amostral. Além disso, a avaliação a qualidade de vida das mulheres também poderia avaliada para ver possíveis relações. O estudo apresenta como implicações práticas/clínicas, subsídios para tomadas de decisões de fisioterapeutas que atuam com a saúde da mulher, promover estratégias, ações e intervenções de promoção da saúde para mulheres mastectomizadas no contexto da interdisciplinaridade.

CONCLUSÃO

Houve uma alta prevalência de disfunção sexual entre as mulheres mastectomizadas. Em relação aos domínios da resposta sexual que se mostraram alteradas foram: excitação, lubrificação, orgasmo, satisfação e dor neste grupo. Sugerimos, portanto, que novos estudos que abordem essa temática sejam realizados, para elucidar a relação entre os domínios da resposta sexual e a DSF em mulheres submetidas à mastectomia.

\section{REFERÊNCIAS}

1. Bastianello MR, Hutz CS. Otimismo e suporte social em mulheres com câncer de mama: uma revisão sistemática. Psicol Teor Prat. 2016;18(2):19-33.

2. Ministério da Saúde; Instituto Nacional de Câncer- INCA.Biblioteca On-Line. Acesso em: 11 dez. 2010. Disponível em: https://www.inca.gov.br/controle-do-cancer-demama/conceito-e-magnitude

3. Fagundes, MKV Vaccaro MM. Mastectomia radical e sua influência sobre a vivência da sexualidade feminina. Uningá Review. 2016; 25(1):79-86.

4. Cesnik VM, Santos MA. Mastectomia e sexualidade: uma revisão integrativa. Psicologia: Reflex Crit. 2012;25(2):339-49.

5. Almeida TRD, Guerra MR, Filgueiras MST. Repercussões do câncer de mama na imagem corporal da mulher: uma revisão sistemática. Physis. 2012;22(3):1003-29.

6. Auwad WA, Hagi SK. Female sexual dysfunction: what Arab gynecologists think and know. Int Urogynecol J. 2012;23(1):919-27.

7. Santos DBS, Santos MA, Vieira EM. Sexualidade e câncer de mama: Uma revisão sistemática da literatura. Saúde Soc. 2014;23(4):1342-55.

8. Raposo JV, Moreira TL, Arbinaga F, Teixeira CM. Satisfação sexual em pacientes com câncer. Acta Colomb Psicol. 2017;20(2):84-94.

9. Macedo JB, Brondani AS, Costa GS, Messias BES, Nardi LLD, Braz MM. Occurrence of sexual dysfunctions in mastectomized females with or without breast reconstruction. Acta Scientiarum. 2018;40(1):e34544.

10. Antunes MD, Nishida FS. Morbidade hospitalar em idosos do paraná durante o ano de 2016. 


\section{CONFLITO DE INTERESSES}

Os autores declaram não haver conflitos de interesse

\section{AUTOR PARA CORRESPONDÊNCIA}

\section{Mateus Dias Antunes}

R. Cipotânea, 51 - Vila Butantã, 05360-160 São Paulo - SP, Brasil.

E-mail: mateusantunes@usp.br 1-7.

13. Sreelakshmy $K$, Velayudhan $R$, Kuriakose $D$, Nair R. Sexual dysfunction in females with depression: a cross- sectional study. Trends Psychiatry Psychother. 2017;39(2):106-9.

14. Pegorare ABGS, Silveira KR, No Ana PS, Barbosa SRM. Assessment of female sexual function and quality of life among breast cancer survivors who underwent hormone therapy. Mastology. 2017;27(3):237-44.

15. Siegel R, DeSantis C, Virgo K, Stein K, Mariotto $A$, Smith $T$ et al. Cancer treatment and survivorship statistics, 2012. CA Cancer J Clin. 2012;62(4):220-41.

16. Souza ACS. Antidepressivos e disfunções sexuais. Rev Psychiatrv. 2017;17(8):1-10.

17. Siegel R, DeSantis C, Virgo K, Stein K, Mariotto $A$, Smith $T$ et al. Cancer treatment and survivorship statistics, 2012. CA Cancer J Clin. 2012;62(4):220-41.

18. Araújo, A. C. G. C.; Nascimento, C. M. L.; Neto, P. F. A.; Silva,B. R. C.; Araújo, K. Q. M. A.; Gonçalves, A. K. Prevalência de disfunção sexual em mulheres sobreviventes de câncer de mama. Top Cienc Saude. 2019;11(1): 107-16.

19. Berghmans B. Physiotherapy for pelvic pain and female sexual dysfunction: an untapped resource. Inter Urogynecol J. 2018;29(1): 631-38. 